\title{
Electron backscattering from dynamical impurities in a Luttinger liquid
}

\author{
Pablo San-Jose* and Francisco Guined \\ Instituto de Ciencia de Materiales de Madrid, CSIC, Cantoblanco, E-28049 Madrid, Spain. \\ Thierry Martin $\AA^{\ddagger}$ \\ Centre de Physique Théorique, Université de la Méditerranée, Case 907, 13288 Marseille
}

(Dated: February 2, 2008)

\begin{abstract}
Electron backscattering in a Luttinger liquid with an impurity is investigated in the presence of zero point motion of the phonon lattice. The impurity can mean either a mass defect, an elastic defect or a pinning defect. The phonon spectrum is then affected by the presence of the defect, which enters in the renormalization group equations for backscattering. The RG equation becomes dependent on the energy cutoff for finite Debye frequency $\omega_{D}$ giving rise to finite energy effects. We compute the local density of states and show how the renormalization group flow is affected by a finite $\omega_{D}$.
\end{abstract}

\section{INTRODUCTION}

It is well known that electron-electron interactions give rise to Luttinger behavior in one-dimensional metallic systems. Standard studies assume the existence of an instantaneous interaction between electrons. Retardation effects need to be included if the interactions involve excitations, such as phonons, of energies much lower than the electronic bandwidth. An interesting class of materials where such excitations are expected to play a role are the carbon nanotubes [1], where one dimensional features coexist with significant electron-electron and electronphonon interactions.

The effect of the coupling to acoustic phonons on a Luttinger liquid was studied in 2, 3]. An interesting new feature is the existence of the Wentzel-Bardeen instability [4, 5] for sufficiently large couplings. The analysis of the backscattering induced by an impurity on the transport properties of a Luttinger liquid [6] has also attracted a great deal of interest. The extension of this problem to the case of a static impurity interacting with a coupled electron-phonon system is discussed in 7$]$.

In the present work, we generalize previous work to the case where the source of backscattering in a one dimensional system has internal dynamics, and retardation effects have to be taken into account. We consider, in particular, the effect of a local modification of the elastic properties of a Luttinger liquid where the interaction between the electrons and the acoustic phonons is not negligible. We think that this study can be relevant for the carbon nanotubes. The electron-phonon coupling in nanotubes has been estimated to be significant [8], and it is possibly the origin of superconducting features at low temperatures 9, 10]. For sufficiently small radii, the nanotubes are expected to be close to the Wentzel-Bardeen instability [1]]. The repulsive electron-electron interac-

\footnotetext{
*Electronic address: pablo.sanjose@icmm.csic.es

$\dagger$ Electronic address: paco.guinea@icmm.csic.es

‡Electronic address: martin@cpt.univ-mrs.fr
}

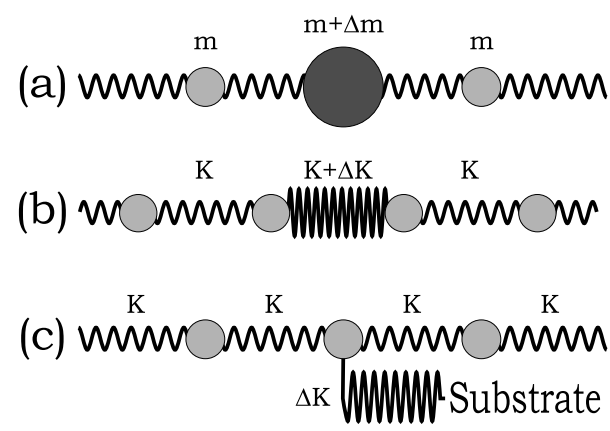

FIG. 1: a) An impurity atom is inserted in the atomic chain, resulting in a mass defect. b) a structural deformation of the lattice results in an elastic defect. c) interaction of one atom with an underlying substrate results in a pinning defect.

tion is also large 12, 13, 14]. Both impurities and phonons are expected to play an important role in the transport properties of nanotubes [15, 16, 17, 18]. Note also that the Luttinger liquid characteristics of carbon nanotubes have been studied by measuring the changes induced by impurities and contacts on the transport properties 19].

The next section discusses the model of the bulk system studied in this paper. The different types of defects considered here are described in section III. The method of calculation used, based on the Renormalization Group approach described in $[6]$ is presented in section IV. A discussion and the main conclusions of the paper can be found in Section V.

\section{THE ELECTRON-PHONON CHAIN.}

Consider a 1-D chain (length $L$ ) of $N$ atoms, mass $m$, each pair separated by $a$ and elastic constant $K$ between each pair. In the absence of inhomogeneities, the action describing the lattice is written in terms of the Fourier components of the lattice deformation field $d_{k, \omega}$ :

$$
S_{p h}=\frac{1}{2} \mu a \sum_{k \omega}\left[\omega^{2}-\omega_{D}^{2} \sin ^{2}\left(\frac{k a}{2}\right)\right] d_{k, \omega} d_{-k,-\omega}
$$


where $\omega_{D}$ is the Debye frequency. The sound velocity is $c=a \omega_{D} / 2=a \sqrt{K / m}$, and $\mu=m / a$ stands for the linear mass density. We will in the following take the continuum approximation $4 \sin ^{2}(k a / 2) / a^{2} \rightarrow k^{2}$.

The charge sector of the electronic degrees of freedom is described as a continuum Luttinger model (note that phonons and lattice deformations do not couple to the spin sector). Using a standard bosonized notation, we have:

$$
S_{e}=\frac{1}{2} \frac{a}{u K_{\rho}} \sum_{k \omega}\left(\omega^{2}-u^{2} k^{2}\right) \phi_{k, \omega} \phi_{-k,-\omega}
$$

Note the analogy between Eqs.(11) and (2) in the limit of long wavelengths. One can identify $\mu$ with $\left(u K_{\rho}\right)^{-1}$ and the sound velocity $c$ with the charge velocity $u$. Usually $u$ is greater than $c$, since it is of the order of $v_{F}$ in most cases, but this need not be the case in the presence of interactions.

We describe the electron-phonon interaction by a deformation potential:

$$
S_{e-p h}=-\frac{a}{u K_{\rho}} b \nu \sum_{k \omega} k^{2} \phi_{k, \omega} d_{-k,-\omega}
$$

where the approximate sign holds for long wavelength excitations. The reduced coupling strength is defined as $b \equiv g \sqrt{u K_{\rho} / \mu}$ and the reduced mass density as $\nu^{2} \equiv$ $\mu u K_{\rho}$.

The complete model can be then be expressed in terms of a diadic Green's function:

$$
S_{0}=\frac{1}{2} \sum_{k \omega}\left[\phi_{-k-\omega}, d_{-k-\omega}\right] \mathbf{G}_{\mathbf{0}}^{-1}(k, \omega)\left[\begin{array}{c}
\phi_{k \omega} \\
d_{k \omega}
\end{array}\right]
$$

where the Green's function of this action then reads after inversion:

$$
\begin{gathered}
\mathbf{G}_{\mathbf{0}}(k, \omega)=\frac{u K_{\rho}}{2 c \omega_{D}} \prod_{\epsilon= \pm}\left(\tilde{\omega}^{2}-\frac{v_{ \pm}^{2}}{c^{2}} \tilde{k}^{2}\right)^{-1} \\
\times\left[\begin{array}{cc}
\tilde{\omega}^{2}-\tilde{k}^{2} & \frac{b c^{2}}{\nu} \tilde{k}^{2} \\
\frac{b c^{2}}{\nu} \tilde{k}^{2} & \frac{1}{\nu^{2}}\left(\tilde{\omega}^{2}-\frac{u^{2}}{c^{2}} \tilde{k}^{2}\right)
\end{array}\right]
\end{gathered}
$$

Here $\tilde{k}=(k a) / 2$ is the dimensionless momentum, $\tilde{\omega}=\omega / \omega_{D}$ is the dimensionless frequency and $v_{ \pm} \equiv$ $\sqrt{\frac{1}{2}\left(c^{2}+u^{2} \pm \sqrt{4 c^{4} b^{2}+\left(c^{2}-u^{2}\right)^{2}}\right)}$ are the group velocities of the polarons (hybridized electron and phonon modes) in the bulk. Note that the Wentzel-Bardeen instability comes about when $v_{-}$becomes imaginary, i.e. for coupling strength $b>u / c$. This completes the description of the homogeneous electron phonon system.

\section{THE DEFECTS.}

\section{A. Local change in the mass density.}

We assume that the defect changes locally both the mass density and the lattice elastic constants. A change in the mass density at position $x_{0}$ is described by the action:

$$
\Delta S_{m}=\frac{1}{2} \int d t \Delta m\left(\partial_{t} d_{x_{0}, t}\right)^{2}=\frac{1}{2} \sum_{\omega} \Delta m \omega^{2} d_{x_{0} \omega} d_{x_{0}-\omega}
$$

This results in a modified Green's function which is no longer translational invariant. In the absence of electronphonon coupling, the phonon Green's function becomes:

$$
\left.G\left(x_{0}, \omega\right)\right|_{22} \stackrel{b=0}{=} \frac{u K_{\rho}}{2 c \omega_{D}} \frac{1}{\nu^{2} \tilde{\omega}^{2} \sqrt{1-1 / \tilde{\omega}^{2}}+\Delta \nu^{2} \tilde{\omega}^{2}}
$$

with the notation $\Delta \nu^{2}=u K_{\rho} \Delta m / a$, which ranges from $\Delta \nu^{2}=-\nu^{2}$ (the mass at position $x_{0}$ is zero) to infinity. The retarded, advanced, and causal Green's functions are obtained by adding a small imaginary part (with the corresponding sign) to $\omega$, and choosing the principal resolution of the square root, in which the branch cut is in the negative real axis.

In the absence of a mass defect, we obtain a simple phonon band for the local density of states. Introducing a finite defect modifies the phonon spectrum. We can describe the changes induced by the defect by the transmission coefficient for phonons of energy $\tilde{w}$. The transmission coefficient at low energies is:

$$
T(\tilde{w})=\frac{1}{1+i \tilde{w} \frac{\Delta m}{m}}
$$

Hence, $\lim _{\tilde{w} \rightarrow 0} T(\tilde{w})=1$. The defect is tranparent for low energy phonons. This result is a consequence of the fact that the defect does not break the translational invariance of the lattice. Hence, the system supports long wavelength phonons of low energy. The deviations from this low energy limit take place at energies $\tilde{w} \sim m / \Delta m$. For massive defects, $\Delta m \gg m$, the transmission coefficient tends to zero at finite, but low, energies. The lattice is effectively divided into two disconnected parts in this range of energies.

The general case $(b \neq 0)$ requires some matrix inversion:

$$
G\left(x_{0}, \omega\right)=\left[G_{0}\left(x_{0}, \omega\right)^{-1}+\left(\begin{array}{cc}
0 & 0 \\
0 & \Delta m \omega^{2}
\end{array}\right)\right]^{-1} .
$$

Analytic expressions can be obtained for all ranges of $\Delta m$. Regardless of the presence of the defect, the electron band and the phonon bands hybridize, as the phonon LDOS shows a peak at frequency $u \omega_{D} / c$, similarly the electron spectrum shows a peak at $\omega_{D}$. The fact that the perturbation enters in eq. (9) multiplied by $\omega^{2}$ is a sign of its irrelevance at low energies, as discussed earlier.

\section{B. Local change in the elastic constants.}

In the case of an elastic defect, one modifies the spring constant between two atoms of the chain $x_{0}$ and $x_{1}$. The 
action associated with this type of defect then reads

$$
\begin{aligned}
\Delta S_{K} & =-\Delta V=-\frac{1}{2} \int d t \Delta K\left(d_{x_{1}, t}-d_{x_{0}, t}\right)^{2}= \\
& =-\frac{1}{2} \sum_{\omega} \Delta K\left[d_{x_{0}-\omega}, d_{x_{1}-\omega}\right]\left(\begin{array}{rr}
1 & -1 \\
-1 & 1
\end{array}\right)\left[\begin{array}{l}
d_{x_{0} \omega} \\
d_{x_{1} \omega}
\end{array}\right]
\end{aligned}
$$

Note that contrary to the case of a mass defect, here two phonon fields (at locations $x_{0}$ and $x_{1}$ ) enter this action. In order to obtain the local density of states, we first derive the $3 \times 3$ local Green's function matrix $\mathbf{G}_{\mathbf{0}}$ associated with fields $\phi_{x_{0}, \omega}, d_{x_{0}, \omega}$ and $d_{x_{1}, \omega}$ in the absence of the perturbation:

$i \mathbf{G}_{\mathbf{0}}=\left(\begin{array}{ccc}\left\langle\phi_{x_{0}, \omega} \phi_{x_{0},-\omega}\right\rangle & \left\langle\phi_{x_{0}, \omega} d_{x_{0},-\omega}\right\rangle & \left\langle\phi_{x_{0} \omega} d_{x_{1}-\omega}\right\rangle \\ \left\langle d_{x_{0}, \omega} \phi_{x_{0},-\omega}\right\rangle & \left\langle d_{x_{0}, \omega} d_{x_{0},-\omega}\right\rangle & \left\langle d_{x_{0}, \omega} d_{x_{1},-\omega}\right\rangle \\ \left\langle d_{x_{1}, \omega} \phi_{x_{0},-\omega}\right\rangle & \left\langle d_{x_{1}, \omega} d_{x_{0},-\omega}\right\rangle & \left\langle d_{x_{1}, \omega} d_{x_{1},-\omega}\right\rangle\end{array}\right)$

which requires integration of the full Green's function of Eq. (5) over momentum. Next, Dyson's equation is used to take the defect into account:

$$
\mathbf{G}=\mathbf{G}_{\mathbf{0}}+\mathbf{G}_{\mathbf{0}} \cdot \Delta \mathbf{V}\left(\mathbf{1}-\Delta \mathbf{V G}_{\mathbf{0}}\right)^{-1} \cdot \mathbf{G}_{\mathbf{0}}
$$

with $\Delta \mathbf{V}$ the potential matrix associated with the action (11):

$$
\Delta \mathbf{V}=\Delta K\left(\begin{array}{rrr}
0 & 0 & 0 \\
0 & 1 & -1 \\
0 & -1 & 1
\end{array}\right)
$$

In the absence of electron-phonon coupling, analytic expressions for the perturbed phonon Green's function for $d_{x_{0} \omega},\left.G\right|_{22}$ are obtained:

$$
\left.G\right|_{22}=\frac{u K_{\rho}}{2 c \omega_{D}} \frac{\left(\frac{K}{\Delta K}+1\right)-2\left(\tilde{\omega}^{2}+\tilde{\omega}^{2} \sqrt{1-1 / \tilde{\omega}^{2}}\right)}{\left(\frac{K}{\Delta K}+1\right) \tilde{\omega}^{2} \sqrt{1-1 / \tilde{\omega}^{2}}+\tilde{\omega}^{2}}
$$

The phonon tansmission coefficient at low energies can be written as:

$$
T(\tilde{w})=\frac{1}{1-i \frac{\Delta K}{K+\Delta K} \tilde{w}^{2}}
$$

When the electron-phonon coupling is finite the imaginary part of the local Green's function can be negative even if $\Delta K$ is within its allowed range. This result is a local counterpart of the Wentzel-Bardeen instability of the extended version. Similar instabilities have been studied in other strongly correlated systems [20].

As in the case of a local change in the mass distribution, a local modification of the elastic constant does not change the value of the transmission coefficient at low energy, as this perturbation does not alter the translational invariance of the system. The range of energies for which the transmission coefficient is close to one is $\tilde{w} \sim \sqrt{K / \Delta K}$.

\section{Pinning of the lattice.}

We can also consider the case where the lattice is pinned by an external perturbation. Then, the action due to this defect is:

$$
\begin{aligned}
\Delta S_{K} & =-\Delta V=-\frac{1}{2} \int d t \Delta K d_{x_{0}, t}^{2}= \\
& =-\frac{1}{2} \sum_{\omega} \Delta K d_{x_{0}-\omega} d_{x_{0} \omega}
\end{aligned}
$$

Using the methods discussed earlier, we find:

$$
G\left(x_{0}, \omega\right)=\left[G_{0}\left(x_{0}, \omega\right)^{-1}+\left(\begin{array}{cc}
0 & 0 \\
0 & \Delta K
\end{array}\right)\right]^{-1} .
$$

In this case the perturbation is relevant at low energies, and the transmission coefficient goes to zero at low energies. The lattice is effectively divided into two decoupled pieces. When the electron-phonon coupling is zero, the phonon transmission coefficient at low energies is:

$$
T(\tilde{w})=\frac{\tilde{w}}{\tilde{w}+i \frac{\Delta K}{2 K}}
$$

Above a crossover energy, $\tilde{w} \geq \Delta K / K$, the defect is transparent to the phonons, and the transmission coefficient approaches one.

\section{ELECTRON BACKSCATTERING.}

\section{A. Flow equations.}

The defects considered in the previous section modify the phonon and electron LDOS. Now we address how each type of defect affects electron backscattering. We consider here the limit of weak backscattering only. Then, without loss of generality, the action corresponding to a short range potential reads:

$$
\begin{aligned}
\Delta S_{e} & =\frac{1}{4} \delta_{e} \sum_{r s} \int d \tau \Psi_{r s x_{0} \tau}^{+} \Psi_{-r s x_{0} \tau}=\ldots= \\
& =\delta_{e} \int d \tau \cos \left(\sqrt{2 \pi} \theta_{\rho x_{0} \tau}\right) \cos \left(\sqrt{2 \pi} \theta_{\sigma x_{0} \tau}\right)
\end{aligned}
$$

Following [7], the flow of the electron backscattering $\delta_{e}$ induced by the defect obeys the equation:

$$
\frac{2}{\delta_{e}} \frac{d \delta_{e}}{d l}=1-\Lambda\left(\left.\mathcal{G}\right|_{1,1}\left(x_{0}, \Lambda\right)+\left.\mathcal{G}\left(x_{0},-\Lambda\right)\right|_{1,1}\right)
$$

$\Lambda$ is the cutoff that scales as $\Lambda=e^{-l} \Lambda_{0}$, and $\mathcal{G}\left(x_{0}, \omega\right)$ is the Matsubara Green function, which results from performing a Wick's rotation on the action. After the Wick's rotation, using the a path integral formalism, the weight of the paths becomes $e^{-\mathcal{S}}$ instead of $e^{i S}$, where $\mathcal{S}$ is $\mathcal{S}=$ $\sum_{k \omega}[\phi, d] \mathcal{G}^{-1}[\phi, d]^{\dagger}$ instead of $\mathcal{S}=\sum_{k \omega}[\phi, d] G^{-1}[\phi, d]^{\dagger}$ 

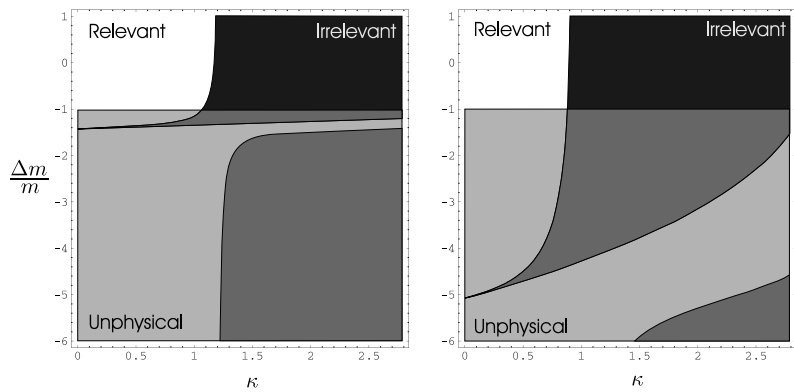

FIG. 2: Backscattering flow diagram for a mass defect and two different values of the cutoff, $\Lambda=\omega_{D}$ (left) and $\Lambda=$ $0.2 \omega_{D}$. This time the lower grey zone is unphysical, since it corresponds to a negative substitute mass. This time the defect resonance always remains in this region.

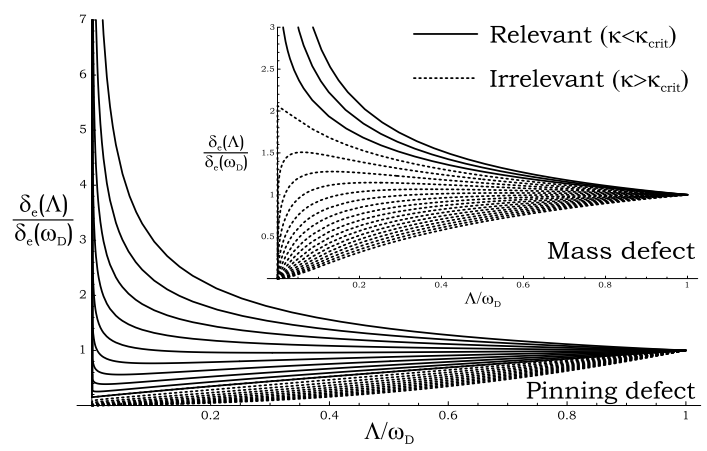

FIG. 3: Flow diagrams from a given initial backscattering term as function of energy. Top: defect which changes locally the mass with $\Delta m=1$. Bottom: defect which pins locally the lattice, with $\Delta K=0.1$. Different curves correspond to different $\kappa$ electron interaction parameters. Velocities were chosen to be $u=2 c$ and $b$ was set to a constant $2 \%$ below the Wentzel-Bardeen instability.

(where $G$ was chosen in the causal prescription for convergence). With these definitions it can be shown that $\mathcal{G}(k, \omega)=-G(k, i \omega)$. This quantity is real, due to the fact that $\mathcal{G}(k,-\omega)=\mathcal{G}(k, \omega)$.

The main difference between the flow of the backscattering term in eq.(20) and the flow associated with elastic scattering in Luttinger liquids $[\underline{6}]$ is the non trivial cutoff dependence in the right hand side in eq. (20). Retardation effects can also been induced by elastic defects in systems of electrons coupled to phonons [7] due to the difference between the Debye energy, $\omega_{D}$, and the electronic bandwidth. If the defect changes the elastic properties of the lattice, a different crossover at energies lower than $\omega_{D}$ is also possible, as discussed in section IIIB.

At the lowest energies or temperatures, a local change in the elastic properties or the mass density does not affect the phonon transmission coefficient. At these scales, the defect plays no role, and the scaling of the backscattering term is given by the results in [7]. At higher energies or temperatures, although they can be small compared to $\omega_{D}$, the phonon transmission coefficient can be significantly reduced, more so the closer the system is
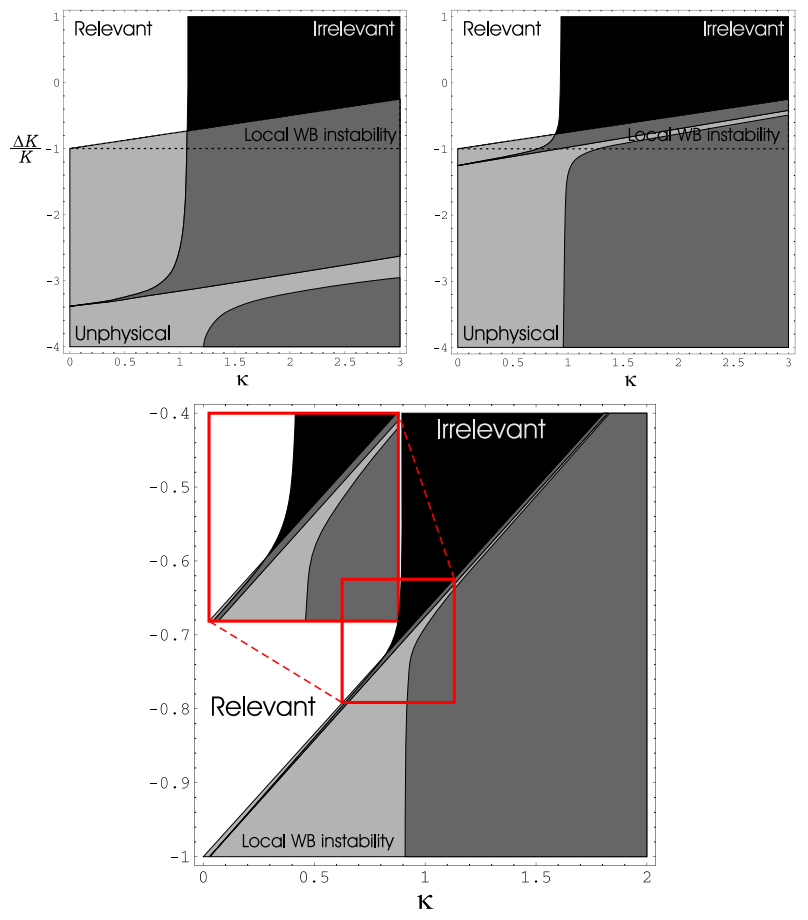

FIG. 4: Backscattering flow diagram for an elastic defect and three different values of the cutoff, $\Lambda=\omega_{D}$ (left), $\Lambda=0.2 \omega_{D}$ (right) and the case of very small cutoff $\Lambda=0.01 \omega_{D}$ (lower plot). The white and black regions denote relevant and irrelevant flow of the backscattering respectively, while in the lower grey zone the system becomes unstable. The local instability boundary (dashed region in the diagram) corresponds to $\Delta K / K<\Delta K_{\text {crit }} / K=-\left(\frac{v_{+} v_{-}}{u c}\right)^{2}$. In the lower plot we have emphasized the irrelevant backscattering sliver responsible for the effective suppression at finite energies of elastic defect backscattering close to $\Delta K_{\text {crit }}$.

to the bulk Wentzel-Bardeen instability. As the quasiparticles are made up of a combination of electron and phonon modes, this effect tends to enhance the electronic backscattering.

\section{B. Mass defects}

The sign of the flow of the backscattering term in eq.(20) allows us to divide the parameter space into regions where the flow is renormalized towards higher values (relevant) or towards lower values (irrelevant) at a given energy. These regions are shown in Fig. 20 for a mass defect (see section IIIA). The separatrix between the two regions tends to be a straight line, independent of $\Delta M$ at low energies, in agreement with [7], where a case equivalent to $\Delta M=0$ was considered. At higher energies, the region where the backscattering term appears to be relevant is enlarged. Hence, the flow of the backscattering term is not homogeneous in the regions where the right hand side of eq. (20) changes sign as function of energy. The flow of a given initial backscattering term is 


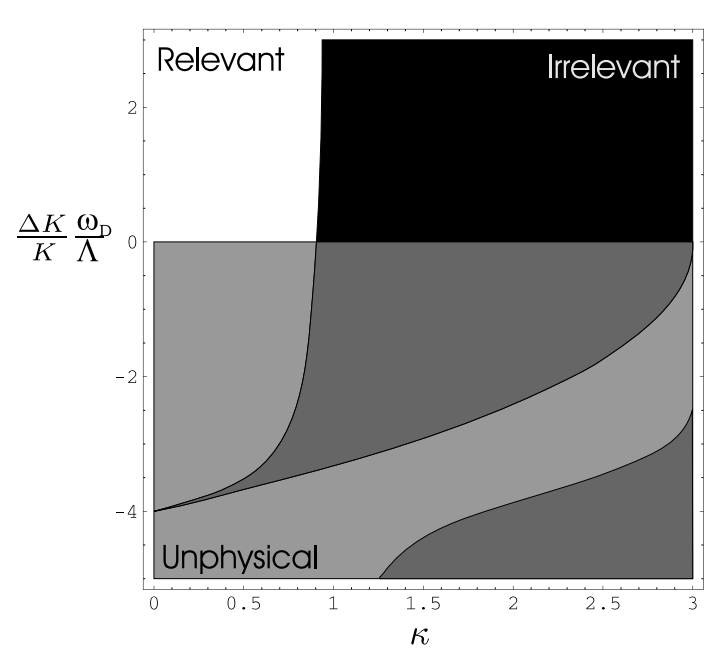

FIG. 5: Backscattering flow diagram for a defect which elastically pins the lattice at a given site (due e.g. to the interaction with a substrate).

shown in the upper part of Fig. 3]. The relevant flow is monotonous, although it deviates significantly from an exponential dependence on energy [6] near the critical line. The irrelevant region of the paremeter space, which is $\kappa>\kappa_{\text {crit }}=\frac{v_{+} v_{-}\left(v_{+}+v_{-}\right)}{\left(c^{2}+v_{+} v_{-}\right) u}$ is non-monotonous close to the WB instability, giving an enhanced backscattering of electrons when we are closed to the $\kappa_{\text {crit }}$ boundary.

\section{Elastic defects}

The flow of the backscattering term is similar when the defect changes the elastic constants of the lattice (see section IIIB). The main difference is that a local WentzelBardeen instability can take place even when the modified elastic constants are positive, and the bulk is stable. The boundary of the locally unstable region is given by $\frac{\Delta K}{K}<\frac{\Delta K_{\text {crit }}}{K}=-\left(\frac{v_{+} v_{-}}{u c}\right)^{2}=-1+\left(\frac{b}{u / c}\right)^{2}$, and is marked by a dashed boundary in Fig. 4. Near this $\Delta K_{\text {crit }}$ line we find a narrow sliver where the electronic backscattering term decreases at high energies, although it can eventually be relevant at low energies. This non monotonous behavior is the inverse of the one discussed for a mass defect. The asymptotic $\Delta \rightarrow 0$ boundary for relevant-irrelevant behavior of backscattering on elastic defects is correctly derived in [7], and it is exactly the same as the $\kappa_{\text {crit }}$ of the mass defect. However at finite temperature and $\kappa<\kappa_{\text {crit }}$ (repulsive electrons) backscattering from such defects can be strongly suppressed when we are close to $\kappa_{\text {crit }}$ from below and to the local WB instability at $\Delta K_{\text {crit }}$. The experimental relevance of this is greater than the bulk Wentzel-Bardeen instability, since the condition $\Delta K \Delta K_{\text {crit }}$ is much more easily achieved than the bulk instability, and can have measurable effects on conductance at finite temperatures. Typical flow diagrams of backscattering amplitude for a given

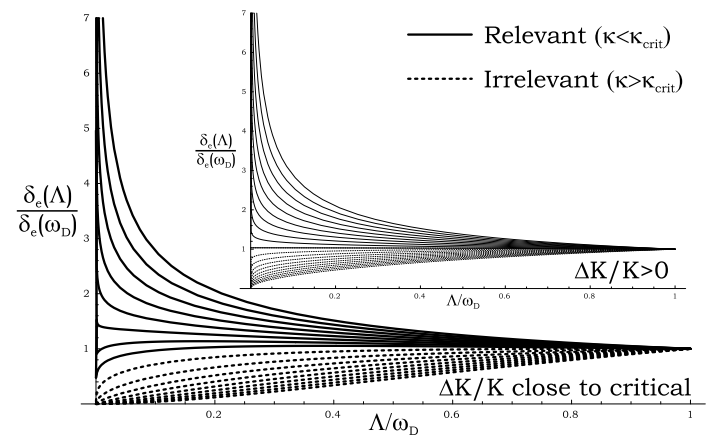

FIG. 6: Flow diagrams from a given initial backscattering term as function of energy. The defect changes locally the elastic constant. In the inset $\Delta K>0$ while in the main plot $\Delta K$ was set to a negative values closer than $1 \%$ to the critical $\Delta K$ which is $\Delta K=-0.4375$ for the parameters used. Velocities are $u=2 c$ and $b=1.5$ is a constant for all curves.

initial value as function of energy and for different bulk parameters are given in Fig. 6]. One can clearly see how the non monotonous behavior discussed above is strongly enhanced near the critical line (main figure).

\section{Pinning of the lattice.}

A representative phase diagram for the case when the lattice is pinned by a defect which breaks translational invariance is shown in Fig. [5]. In this case, and at finite frequencies, the relevant region is reduced, giving rise to a non-monotonous renormalization of the backscattering amplitude that can be suppressed in a similar way as a mass defect could be enhanced at finite frequencies. This effect, as in the case of a substitute mass, is stronger the closer the system gets to the bulk instability. The flow of a given initial backscattering term is shown in the lower part of Fig. 3 for a system close to the Wentzel-Bardeen instability. Note moreover that the pinning defect case presents a peculiar scaling of the flow equations, which only depend on $\Delta_{K} / \Lambda$ (see vertical axis of Fig. [5]).

\section{CONCLUSIONS.}

We have analyzed the role of lattice defects in Luttinger liquids with significant interactions between electrons and acoustical phonons. The quasiparticles of the system are combinations of electron-hole pairs and phonons. A lattice defect modifies locally the propagation of the phonons, inducing a change in the electronic transport properties as well.

A lattice defect which does not break the translational invariance of the lattice is irrelevant at low energies, as it is transparent to low frequency phonons. The borderline which separates the regions where electronic backscattering is relevant or irrelevant is not affected by a defect of this type, and depends only on bulk parameters [7]. There 
is a range of energies, which can be much lower than the Debye frequency, $\omega_{D}$, where the defect scatters the phonons strongly. In this region of energies or temperatures, the electronic backscattering can be significantly enhanced. Thus the flow of an electronic backscattering term can be non monotonous, unlike the case of a Luttinger liquid with elastic scattering [ $[\underline{6}]$, giving rise to measurable conductance dependence on temperature, for example. Even in a system with attractive interactions, a mass defect can block effectively the transport over a significant range of temperatures. On the other hand in a system with repulsive electronic interactions an elastic defect that is close enough to the local Wentzel-Bardeen instability can prove to be unexpectedly transparent to electrons. This local instability can be induced by a strong enough local softening of the elastic constant of the chain in the presence of electron-phonon interactions.

\section{ACKNOWLEDGEMENTS.}

We are thankul to J. González for many helpful comments. P. S. J. and F. G. acknowledge financial support from MCyT (Spain) through grant MAT2002-0495-C0201.
[1] M. S. Dresselhaus and P. C. Eklund, Adv. in Phys. 49 (2000).

[2] D. Loss and T. Martin, Phys. Rev. B 50, 12160 (1994).

[3] T. Martin and D. Loss, Int. J. Mod. Phys. B 9, 495 (1995).

[4] G. Wentzel, Phys. Rev. 83, 168 (1951).

[5] J. Bardeen, Rev. Mod. Phys. 23, 261 (1951).

[6] C. L. Kane and M. P. A. Fisher, Phys. Rev. Lett. 68, 1220 (1992).

[7] T. Martin, Physica D 83, 216 (1995).

[8] J. V. Álvarez and J. González, Phys. Rev. Lett. 91 (2003).

[9] M. Kociak, A. Y. Kasumov, S. Guéron, B. Reulet, I. I. Khodos, Y. B. Gorbatov, V. T. Volkov, L. Vaccarini, and H. Bouchiat, Phys. Rev. Lett. 86, 2416 (2001).

[10] J. González, Phys. Rev. Lett. 87 (2001).

[11] A. D. Martino and R. Egger, Phys. Rev. B 67 (2003).

[12] C. Kane, L. Balents, and M. P. A. Fisher, Phys. Rev.
Lett. 79, 5086 (1997).

[13] R. Egger and A. Gogolin, Eur. Phys. J. B 3, 781 (1998).

[14] R. Egger, Phys. Rev. Lett. 83, 5547 (1999).

[15] B. Reulet, A. Y. Kasumov, M. Kociak, R. Deblock, I. I. Khodos, Y. B. Gorbatov, V. T. Volkov, C. Journet, and H. Bouchiat, Phys. Rev. Lett. 85, 2829 (2000).

[16] D. Bozovic, M. Bockrath, J. H. Hafner, C. M. Lieber, H. Park, and M. Tinkham, Appl. Phys. Lett. 78, 3693 (2001).

[17] D. Bozovic, M. Bockrath, J. H. Hafner, C. M. Lieber, H. Park, and M. Tinkham, Phys. Rev. B 67 (2003).

[18] A. Javey, J. Guo, M. Paulsson, Q. Wang, D. Mann, M. Lundstrom, and H. J. Dai, Phys. Rev. Lett. 92 (2004).

[19] M. Bockrath, D. H. Cobden, J. Lu, A. G. Rinzler, R. E. Smalley, L. Balents, and P. L. McEuen, Nature 397, 598 (1999).

[20] C. C. Wu and P. W. Anderson, Phys. Rev. B 29 (1984). 